\title{
The impact of using musculoskeletal ultrasound imaging and other influencing factors on medication adherence in patients with rheumatoid arthritis: a qualitative study
}

\author{
This article was published in the following Dove Press journal: \\ Patient Preference and Adherence \\ 16 June 2016 \\ Number of times this article has been viewed
}

\author{
Kanta Kumar ${ }^{1,2}$ \\ Karim Raza ${ }^{3,4}$ \\ Paramjit Gill' \\ Sheila Greenfield' \\ 'Primary Care Clinical Sciences, \\ University of Birmingham, Birmingham, \\ ${ }^{2}$ Faculty of Medical and Human \\ Sciences, University of Manchester, \\ Manchester, ${ }^{3}$ Institute of Inflammation \\ and Ageing, University of Birmingham, \\ ${ }^{4}$ Sandwell and West Birmingham \\ Hospitals NHS Trust, Birmingham, UK
}

Correspondence: Kanta Kumar Primary Care Clinical Sciences, University of Birmingham, Vincent Drive, Birmingham BI5 2TT, UK

Email k.kumar@bham.ac.uk
Background: Medication can ease symptoms and limit disease progression in rheumatoid arthritis (RA). Despite this, nonadherence to medication is common in RA. We explored the determinants of high and low adherence to disease-modifying antirheumatic drugs (DMARDs) in patients with RA and provide suggestions on approaches to improving adherence to DMARDs.

Methods: Patients with RA were identified from those who had previously participated in a questionnaire measuring levels of medication adherence. Twenty patients participated (ten high and ten low adherers, as determined by responses to the Medication Adherence Report Scale). In-depth individual semistructured interviews were undertaken until data saturation was reached. Interviews were transcribed and analyzed using a constant comparative method.

Results: Four main themes related to adherence were identified: 1) symptom severity; 2) illness perception; 3 ) perceived benefits and risks of DMARDs; and 4) the quality and quantity of information about RA and DMARDs. In addition, patients' suggestions about strategies to optimize adherence to DMARDs were captured and they fell within the following themes: 1) musculoskeletal ultrasound to explain the disease process and to provide objective feedback about the extent to which their disease activity is being effectively controlled; 2) better explanations of the consequences of poorly controlled RA; and 3) a good relationship with the health professional.

Conclusion: Patients' beliefs about medicines, perceptions about RA, and level of satisfaction with information about DMARDs influenced their adherence to DMARDs. The use of musculoskeletal ultrasound to image the inflamed joint may help to improve patient adherence to DMARDs.

Keywords: rheumatoid arthritis, medication adherence, patients' suggestion to improve medication, disease-modifying antirheumatic drugs

\section{Introduction}

Rheumatoid arthritis (RA) is a chronic inflammatory disease characterized by synovitis and associated with joint destruction. ${ }^{1}$ RA causes progressive decline in functional ability and limits participation in social and work-related activities. ${ }^{2}$ RA is also associated with an increased risk of extra-articular complications including ischemic heart disease. ${ }^{3}$ While curative therapies are not currently available, treatments with disease-modifying antirheumatic drugs (DMARDs) and biologic agents improve joint function, ${ }^{1}$ work productivity, ${ }^{4}$ and health-related quality of life, reducing 
the risk of joint destruction, disability, and morbidity and improving the life expectancy. ${ }^{5}$ Despite these treatments, many patients fail to achieve optimal disease control, in part, due to nonadherence. ${ }^{6}$

The World Health Organization defines adherence as "the extent to which the patient's behavior coincides with the health professional's advice". ${ }^{7}$ Nonadherence to medication remains a major problem across the spectrum of chronic diseases. ${ }^{8}{ }^{8}$ Factors associated with poor medication adherence are complex. ${ }^{7}$ In RA, only a small proportion of the variance in adherence is explained by demographic characteristics which are largely unchangeable, ${ }^{10}$ therefore necessitating the identification of other modifiable factors that could be changed. ${ }^{11,12}$ It is widely reported that patients' beliefs about medicines can have a major impact on their decision to take them. ${ }^{13-15}$

Medication-taking behavior might be enhanced by a better understanding of the types of belief that are associated with poor adherence. ${ }^{16}$ This understanding can be obtained by employing the Necessity-Concerns Framework $(\mathrm{NCF})^{16}$ and the Self-Regulatory Model (SRM)-Common Sense Model (CSM). ${ }^{17}$ The SRM-CSM/NCF framework allows researchers to see how adherence is associated with the ways in which patients judge their personal need for their prescribed medicines relative to their concerns about possible side effects. ${ }^{11,15}$ Studies across long-term conditions including RA have demonstrated the utility of the SRM-CSM/NCF with nonadherence related to doubts about personal need for treatment and concerns about side effects. ${ }^{11,15}$ Patients' judgment about prescribed medications may be influenced by more general attitudes to medicines as a whole, for example, perceptions that medicines are harmful, addictive, and poisonous and are overused by doctors. ${ }^{18}$ Negative attitudes and beliefs about DMARDs appear more prevalent within certain ethnic groups including the South Asian population where many patients have negative attitudes toward DMARDs. ${ }^{19}$ However, those studies that report data on the impact of beliefs about medicines on adherence have not included South Asian patients with RA. ${ }^{13-15,20}$ Health care professionals managing patients with RA need to understand the influence of health beliefs on medication adherence, as nonadherence is costly. ${ }^{10}$ As part of a questionnaire study examining determinants of medication adherence in patients of White British and South Asian origin, ${ }^{21}$ we explored the views about DMARDs and the determinants which influence medicine-taking by patients with RA. In addition, we documented patients' suggestions about adherence-enhancing strategies.

\section{Materials and methods}

Ethical approval for this study was granted by the South Birmingham Research Ethics Committee, and all patients gave written informed consent.

The study took place at Sandwell and West Birmingham Hospitals NHS Trust and University Hospitals Birmingham NHS Foundation Trust, UK. Patients were selected from those who had participated in a previous questionnaire study of 180 patients with RA. ${ }^{21}$ Patients in that cross-sectional study had had a diagnosis of RA (according to the 1987 American College of Rheumatology criteria) ${ }^{22}$ for at least 3 months, were aged 18 and over, and were taking at least one DMARD or had taken a DMARD in the past.

Patients were selected purposively to ensure a mixture of age, sex, language spoken, and employment status (Table 1). ${ }^{21}$

All 180 patients who had taken part in the questionnaire study were given a questionnaire (Medication Adherence Rate Score $)^{15}$ that measured self-reported medication adherence. "High" adherence was defined as a Medication Adherence Rate Score ${ }^{15}$ in the upper tertile (Table 1) and "low" adherence as a score in the lowest tertile. Patients in the middle tertile were excluded. There were 50 high-adhering patients (White British 33, South Asian 17) and 62 low-adhering patients (White British 23, South Asian 39); and from these 112 patients, 36 were invited by mail for an interview. Patients who responded to letters were recruited into the study (high adherers: ten White British, eight South Asians; low adherers: six White British, 12 South Asians); 16 declined.

Table I Demographic data of the patients interviewed

\begin{tabular}{|c|c|c|}
\hline & High adherers* & Low adherers* \\
\hline & $n=10$ & $n=10$ \\
\hline \multicolumn{3}{|l|}{ Sex } \\
\hline Male & 3 & 3 \\
\hline Female & 7 & 7 \\
\hline \multicolumn{3}{|c|}{ Language spoken during interviews } \\
\hline English & 9 & 6 \\
\hline Punjabi & 1 & 4 \\
\hline \multicolumn{3}{|l|}{ Employment } \\
\hline Employed & 4 & 4 \\
\hline Unemployed & 1 & \\
\hline Homemaker & 2 & I \\
\hline Retired & I & 3 \\
\hline Off work due to RA & 2 & 1 \\
\hline
\end{tabular}

Notes: High adherence defined as a MARS of $\geq 25$ (ie, in the upper tertile of responses) and low adherence as a MARS of $\leq 25$ (ie, in the lower tertile of responses). $* \geq 25$ is the maximum possible score of MARS and 18 was the lowest observed score of MARS in our cross-sectional study.

Abbreviations: MARS, Medication Adherence Rate Score; RA, rheumatoid arthritis. 
- What do you think about your medications (DMARDs) that you take for controlling your RA?

- How necessary do you feel the medication (name of DMARD) is for you? (Prompts: do you stop them, how long, would there be a reason for stopping DMARD?)

- Do you feel that these are working to control your symptoms? (Prompt: do you change the doses?)

- Do you have any concerns about the DMARDs?

- Tell me about RA. Do you view this to be a short-term disease or lasting for a long time? (Prompt: do you think DMARD will control your RA?)

- Have you had enough information about your condition and the DMARD?

- How can we improve the area of medicine-taking? What can be done to help patients understand the DMARDs we give? (Themes that arose from the high-adhering patients were introduced to the low-adhering patients.)

Figure I Topic guide based on SRM-CSM and NCF (questions asked during the interviews).

Abbreviations: CSM, Common Sense Model; DMARDs, disease-modifying antirheumatic drugs; NCF, Necessity-Concerns Framework; RA, rheumatoid arthritis; SRM, Self-Regulatory Model.

A group of Patient Research Partners were part of the steering committee that helped with the development of the topic prompt (Figure 1). The topic guide was organized around questions broadly based on the NCF, SRM, ${ }^{14}$ and patient satisfaction with information about DMARDs. Initial questions were general (eg, "What do you think about your DMARDs?"), and were followed by prompts based on the NCF (eg, "How necessary do you feel the DMARD(s) is for you?" and "Do you have any concerns about DMARDs?"). In addition, questions were asked about the patients' adherence or nonadherence to DMARDs, including stopping and changing the doses of DMARDs and reasons for doing so. Patients' recommendations as to how adherence could be improved were also elicited.

Individual interviews with patients were conducted at two hospitals in areas designated for research, away from their routine clinical setting. The interviews were undertaken by KK who was fluent in both English and Punjabi. Five patients chose to be interviewed in Punjabi. Recruitment and interviewing continued until data saturation had been reached in both groups.

Each interview lasted approximately 1 hour. All interviews were audio-taped and transcribed verbatim by KK. A selection of English transcriptions was independently checked for emerging themes by KK, PG, and SG. A sample of the English-translated transcripts from Punjabi-speaking patients was validated for accuracy by an independent health professional (AJ). Any discrepancies regarding themes were discussed and agreed. The overall analysis was organized by labeling data relevant ${ }^{23}$ to each of the questions in the topic guide and then grouping similar themes. ${ }^{24}$ Constant comparison ${ }^{25}$ was used to analyze the transcripts line by line, comparing the themes related to high- and low-adhering patients' views about DMARDs. ${ }^{25}$ Particular emphasis was placed on comparing determinants influencing medicine-taking that differed between ethnic groups. Data on strategies that were recommended by high-adhering patients to help improve the area of medication adherence for patients with RA were identified.

\section{Results}

Twenty interviews were conducted (with ten high-adhering patients and ten low-adhering patients). Fourteen participants were female and eight were in full-time employment. Fifteen were interviewed in English and five in Punjabi. Four themes related to adherence were identified in both high- and lowadhering patients: 1) symptom severity; 2) illness perception; 3) perceived benefits and risks of DMARDs; and 4) the quality and quantity of information about RA, DMARDs, and the need for DMARDs. In addition, patients' suggestions about strategies to optimize adherence to DMARDs were captured (Table 2) and they fell within the following themes: 1) visual representations to explain the disease process and to provide objective feedback about the extent to which their disease activity is being effectively controlled; 2 ) better explanations of the consequences of poorly controlled RA; 3 ) a good relationship with the health professional; and 4) access to patient support groups. Each theme is illustrated with quotations from the transcripts. The data from high-adhering patients are presented first.

\section{Results from patients with high adherence Symptom severity}

Seven patients perceived RA medication to be very important; this was related to patients' current experience of symptoms and their recollection of severe symptoms at disease onset (Quote 1). They were also aware that uncontrolled RA could lead to future health problems (Quote 2). As a result of both symptom severity and awareness of future risks, the high-adhering patients viewed RA medicines to 
Table 2 Comparison of themes and subthemes by adherence level

\begin{tabular}{lll}
\hline Themes & High adherers & Low adherers \\
\hline Symptom severity & - Severe symptoms & - Mild symptoms \\
- Family history of RA & - Patients seek for a cure of RA through their \\
Illness perception & - Patients accept that there is no cure for RA & - Patients accept that RA is a long-term disease \\
& - Patients' beliefs about the necessity of DMARDs & - Patients' concerns about DMARDs outweigh \\
Perceived benefits and & outweigh their concerns & their beliefs about the necessity of DMARDs \\
risks of DMARDs & - Patients' experiences of the beneficial effects & \\
& - Satisfaction with information/visual aids & - Lack of information, no experience of visual aids \\
The quality and & - Good relationship with health professionals & - Poor relationship with health professionals \\
quantity of information & - Visual representations to explain the disease process and to provide objective feedback about the extent to \\
& - Bhich their disease activity is being effectively controlled & \\
Patients' suggestions & - A good relationship with the health professional & \\
about strategies to & - Access to patient support groups & \\
optimize adherence & to DMARDs & Poor knowledge about disease parameters
\end{tabular}

Abbreviations: DMARDs, disease-modifying antirheumatic drugs; RA, rheumatoid arthritis.

be important. Family history of RA appeared to increase the level of importance in four of the patients. (Quote 3).

Quote 1: I will take this medication for the rest of my life. I wouldn't stop taking them just to test it out. I just don't want to go where I was. [59, retired, English speaking, White patient]

Quote 2: I have no doubt in my mind that if I stop taking these medications then I know that I would be in a wheelchair. [34, nurse, English speaking, Asian patient]

Quote 3: I have had experience of my mother. She had exactly the same problem as me. She really suffered. I saw her disease develop over time. That really put the fright in me. [34, nurse, English speaking, Asian patient]

\section{Illness perception}

Regarding patients' views about RA, all ten high-adhering patients identified a range of factors that they felt had caused their RA, but none believed that the disease was a transient illness or was curable. They all accepted the need for long-term therapy (Quote 4) and held the belief that taking DMARDs regularly would help to control the disease (Quotes 5 and 6).

Quote 4: I doubt it very much that it can be cured yes it can be controlled. [62, retired, English speaking, White patient]

Quote 5: When I went to my first appointment with the nurse and the way she was talking it seemed I would be on methotrexate for very long time don't know ... may be even for the rest of life because there isn't a cure for this is there? [52, retired, English speaking, White patient]

Quote 6: I have watched my cousin with this arthritis after twenty years he still has it you know ... so I know it's not going to go away. [51, off work due to RA, English speaking, Asian patient]

\section{Perceived benefits and risks of DMARDs}

Nine patients described their DMARD(s) as working to control their RA. Patients made decisions by weighing up the benefits and concerns and/or perceptions about the efficacy of treatment. For example, those who were taking anti-tumor necrosis factor (TNF) therapy (six patients) believed that their lives had been transformed through taking this class of drug and that it was more effective in controlling their symptoms than their previous nonbiologic DMARDs. This view emerged from both White British and South Asian patients (Quotes 7 and 8). The patients who viewed their medicines as necessary (all ten patients) expressed fewer concerns about medicines and did not question the necessity of DMARDs (Quote 9). This appraisal was based on the theme related to symptom severity and views about control rather than cure. Five patients gave examples of their past experiences of stopping medication and how the decision to discontinue DMARDs for a while had led to disease flares (Quotes 10 and 11). The remaining five gave examples of the persistent presence of RA symptoms. These evaluations helped to form beliefs about the necessity of DMARDs 
and these patients reported that they were now completely adherent to DMARDs.

Quote 7: Rituximab has transformed my life now. The rheumatoid arthritis has kept under control since I have started on that. [62, retired, English speaking, White patient]

Quote 8: Initially I couldn't even bend my knees I couldn't do my household chores, since I have been on methotrexate .... Yes I can do these now. I am a paediatric nurse and I am on my feet all the time. So for me to take the medicines is very important. [36, manager, English speaking, Asian patient]

Quote 9: I think that sometimes you think I am taking all these medicines ... like methotrexate they could damage your kidneys your liver ... but I would rather have that danger rather than how I was before. So the dangers of the drugs that I hear mean nothing to me because I want to be how I am now. [52, house wife, English speaking, White patient]

Quote 10: I stopped it for a while [methotrexate] but my flare had come back so I knew I had to take it. [36, manager, English speaking, Asian patient]

Quote 11: I know this would get worse you see. It's there all the time and stopping the medication will make it worse. [42, machine operator, English speaking, White patient]

A range of concerns about DMARDs appeared to be related to the different types of DMARDs used. For example, concerns about short- and long-term side effects of their current DMARDs and their previous experiences of side effects were mentioned by all ten patients. Three patients expressed the view that taking DMARDs was like taking poison and viewed these drugs to be "unnatural". One South Asian patient viewed methi (an Indian herb) and ginger to be more natural than DMARDs (Quote 12). However, despite these concerns, the drivers for the need for DMARDs led the patients to take their DMARDs as prescribed (Quote 13). Three of the patients expressed that DMARDs were less serious than some other therapies such as certain cancer treatments (Quotes 14-16) and this promoted adherence to DMARDs.

Quote 12: I do try and take methi (Indian herb) and ginger. I have fresh ginger every day to help get rid of the toxins (swelling in joints). [51, off work due to RA, Punjabi speaking, Asian patient]
Quote 13: I really hate this medication [methotrexate]. But I appreciate that I need to take it I guess it's a reasonable compromise. [62, retired, English speaking, White patient]

Quote 14: I thought look ... there are people on cancer medicine where their hair falls out and I am not that bad to take these. [42, machine operator, English speaking, White patient]

Quote 15: You know, at least I haven't got cancer its only arthritis. [52, house wife, English speaking, White patient]

Quote 16: I mean at least it wasn't leukaemia or something, you know. I mean touch wood I mean it's not bad as that. I mean it is bad but you know ... cancer is a bad thing to have. [55, unemployed, English speaking, White patient]

\section{Quality and quantity of information}

When patients were asked about what information they found useful as a basis for their decisions to take DMARDs, they gave a range of spontaneous responses. Eight patients believed that seeing an ultrasound scan of one of their inflamed joints had helped them to understand the nature and extent of their disease. These patients stated that they were less likely to stop taking their DMARDs after having seen imaging evidence of synovitis (Quotes 17-20). The images also helped patients to understand the seriousness of the condition (Quote 21). Two patients made reference to ultrasound scans and plain radiographs as having helped them not only to understand the nature of the disease but also to accept the condition.

Quote 17: Dr XX scanned my joints and he showed me my joints and that helped me understand what was going on in my joints. He explained what was happening ... there were different lights flashing and red was showing swelling in the joints. [42, machine operator, English speaking, White patient]

Quote 18: The scan pictures really helped me to understand the disease and to be honest this really motivates me to take the methotrexate. You see I know why I have got to do it. [36, manager, English speaking, Asian patient]

Quote 19: The scan showed me the lights flashing on my joint swelling. It made me scared and helped me to know how serious the condition can be. [51, off work due to RA, Punjabi speaking, Asian patient]

Quote 20: ... but when she showed me the X-rays I understood the reasons for my pain and also saw the damage that the disease had caused. [55, unemployed, English speaking, White patient] 
Quote 21: When I saw the X-rays and the damage I thought you know ... I need to listen to what is being said and accept this is for life. So I am taking my medication and slowly here I am today not that bad. [34, nurse, English speaking, Asian patient]

Quote 22: It really makes a difference when you can get on with your doctors. Mine explained the disease really well. [42, machine operator, English speaking, White patient]

Quote 23: Yeh I mean it makes it easier when the team support is there it makes you feel that you are not alone and there is support there you know. It does make the journey that much easier otherwise you get lost and we take so many medicines. [36, manager, English speaking, Asian patient]

For some patients (six patients), changes in the measured disease activity parameters (blood tests, disease activity score) produced a positive effect in terms of accepting the need for continuous DMARDs. For others (four patients), a positive view about taking DMARDs was facilitated by a good relationship with health professionals and an in-depth explanation of the nature of the disease (Quote 22), in particular RA severity and long-term consequences and explanations about the treatment options available (Quote 23). Information leaflets and use of the Internet were also identified as useful sources of information (four patients), though others (six patients) stated that the leaflets on RA that they had been given did not effectively communicate the seriousness of the disease and were not helpful in visualizing how untreated synovitis could damage joints. Good explanations regarding the seriousness of the disease, ultrasound scans of joints, support by health professionals, and a good explanation of the fluctuating nature of the disease all acted as factors that motivated patients to begin and continue to take DMARDs.

\section{Results from patients with low adherence \\ Symptom severity}

Some low-adhering patients had similar experiences to high-adhering patients regarding their symptom severity (Quote 24). However, there were five patients (all South Asian) in whom symptom experiences were mild (patient's view) at the beginning and as the treatment gave them relief, their reaction was to stop the medication as they thought that the disease had been cured (Quote 25).

Quote 24: I was in so much pain and I thought gosh I would die rather than staying in bed like that ... it was bad. [44, microbiologist, English speaking, Asian patient]
Quote 25: When I started on methotrexate I was well for about five years. Because I was well for so many years and I didn't really have any pain I made myself believe that the disease has cured. [69, retired, Punjabi speaking, Asian patient]

\section{IIIness perception}

In contrast to high-adhering patients, some of the lowadhering patients held the view that the disease was curable and saw medication as a short-term therapy. This was particularly the case among the South Asian patients (Quotes 26 and 27). Furthermore, their decisions to take medication were based on their perception of RA (Quotes 28 and 29). One South Asian patient had stopped methotrexate to try herbal treatment instead.

Quote 26: For a year I really battled with myself. Even to now this day I believe that the disease can be cured ... it will get better. [33, self-employed, English speaking, Asian patient]

Quote 27: I have been taking them for a year and now you would think that this could be cured ... but it hasn't. [69, retired, Punjabi speaking, Asian patient]

Quote 28: To be honest I thought I am 33 so maybe I am getting old or something. I mean this is only old person disease right ... I mean, for arthritis, people live with it they don't take long-term medicines. [33, self-employed, English speaking, Asian patient]

Quote 29: You see I never heard of arthritis well, I didn't think it would be in a young person like me. So I thought at the time I would be on paracetamol and it would go away. [61, housewife, Punjabi speaking, Asian patient]

\section{Perceived benefits and risks of DMARDs}

Although the low-adhering patients viewed DMARDs as necessary and agreed that these medications were helpful in maintaining their function, they also had concerns. In South Asian patients, fears about dependency led them either to miss doses or to stop treatment temporarily (Quotes 30 and 31). The increased concern about side effects and dependency was partly linked to limited knowledge of the disease; again, this was mainly noted in South Asian patients. Some South Asian patients believed that the long-term usage of DMARDs could lead to additional physiological abnormalities (Quote 32). Three patients stated that medicines were poisonous (Quote 33). Two White British low-adhering patients viewed biologic agents such as anti-TNF to be 
important, but stated that they often missed doses of their nonbiologic DMARDs (Quotes 34 and 35).

Quote 30: I don't like taking them (methotrexate) like the biggest thing for me is the hair loss you know. [44, microbiologist, English speaking, Asian patient]

Quote 31: I know that if I take these everyday then I am dependant on them for the rest of my life. [37, legal secretary, English speaking, Asian patient]

Quote 32: I read that these tablets can give you gastric problems. Then you have to take more tablets it's a never stopping process. [41, off work due to RA, Punjabi speaking, Asian patient]

Quote 33: You see these are not natural. I know I have to take them but they are poisons. When I don't need them I stop them for a while. [61, house wife, Punjabi speaking, Asian patient]

Quote 34: I am on Humira and this keeps my disease under control. I don't think I should keep taking my methotrexate when I am controlled more on this drug [Humira]. [41, social worker, English speaking, White patient]

Quote 35: I will be honest with you ... since I have been on these injections [anti-TNF therapy] I really think back why was I even taking these other ones [sulphasalazine]. [63, retired, English speaking, White patient]

\section{Quality and quantity of information}

The reasons for low adherence to DMARDs were linked to a lack of understanding about RA in six patients (Quotes 36 and 37); this was particularly noted among the South Asian patients. The majority of South Asian patients attributed their poor adherence to DMARDs, in part, to the fact that they felt that RA was not generally well known or taken seriously within the South Asian community (Quote 2). Five South Asian patients held the view that arthritis was a disease of the elderly and they did not understand the need for long-term therapy. Furthermore, the South Asian patients had very little understanding of the fluctuating pattern of the disease (Quote 38). Patients expected more information from their health professionals and felt that they were not always as aware of disease parameters as they would like to be (Quote 39). When asked whether they had experienced having an ultrasound scan of the joints, none of the patients in the lowadhering group had been exposed to this test (Quote 40).
Quote 36: Who cares if I have rheumatoid arthritis? Everyone has joint pains and this what people generally think. [69, retired, Punjabi speaking, Asian patient]

Quote 37: I still believe that this disease is not holding the same awareness as the heart diseases ... so people don't take arthritis seriously ... err the medication is not taken seriously. [37, legal secretary, English speaking, Asian patient]

Quote 38: I didn't know that this disease can come and go like this. If I had known I would not reduce my medications. [33, self-employed, English speaking, Asian patient]

Quote 39: No I wasn't aware of this my doctor didn't tell me [referring to disease activity measure - disease activity score]. So this works like a blood pressure when it is high you know it's serious? [41, off work due to RA, Punjabi speaking, Asian patient]

Quote 40: No I didn't ever have anything like this. [referring to ultrasound scan of joints] [37, legal secretary, English speaking, Asian patient]

\section{Patients' suggestions about strategies to optimize adherence to DMARDs}

Themes that acted as drivers for high adherence to DMARDs (such as the quality and quantity of information provided) were suggested to low-adhering patients as potential ways of improving their adherence to DMARDs. All low-adhering patients (ten patients) agreed on the potential benefits of the drivers that were suggested by high-adhering patients. When patients with low adherence were asked about the types of visualization tool they felt would best improve adherence, they said they would prefer to see real-time ultrasound images rather than static images of joints that were not their own. However, some patients (three patients) indicated that pictorial representations would be better included in leaflet form. Seven low-adhering patients believed that the visualization of swelling in joints would enhance patients' understanding of the nature and severity of the disease and the need to take medicines in the long term (Quotes 41 and 42). All ten patients further added that showing them the results of other disease parameters such as a measure of inflammatory makers in the blood or composite disease activity scores would also encourage them to take their DMARDs as prescribed (Quote 43). All ten patients highlighted that good communication with health professionals, health professional support, and better explanation of the risks of RA to their health would 
all promote better medication adherence (Quotes 44-46). Three patients felt that the level of engagement with disease information was an individual choice (Quote 47).

Quote 41: I think the scan would change your attitude to how you think about medicines .... [44, Microbiologist, English speaking, Asian patient]

Quote 42: You see I need something to validate to show me that I have a condition serious as this (rheumatoid arthritis). I think a scan can do this .... [37, legal secretary, English speaking, Asian patient]

Quote 43: My friend has a sugar monitoring machine he knows what a good and high level is. So if you told patients about disease activity levels it would encourage knowing how the medicines are working. [69, retired, English speaking, White patient]

Quote 44: Knowing how this disease will affect us in the future needs to be explained ... so the seriousness of the disease does need to be told .... [56, off work due to RA, Punjabi speaking, Asian patient]

Quote 45: You see the disease isn't viewed seriously, so if the risks aren't explained then people will not take the medication seriously. [63, retired, Punjabi speaking, Asian patient]

Quote 46: Knowing the team is there to support you ... the journey is shared and if the patients are made to feel like this then it's easier, you know. [69, retired, English speaking, White patient]

Quote 47: Hmm I am not sure on what would help .... I think it doesn't matter what you use to explain the disease; it's all up to the individual, isn't it? I mean if I want to know more then I will, but it's all on how I want to take this .... [63, retired, English speaking, White patient]

\section{Discussion}

This study has highlighted the role of symptom experience, illness perceptions, the perceived benefits and risks of DMARDs, and the quality and quantity of information about RA and DMARDs in determining adherence to DMARDs. The findings provide an insight into some of the drivers and barriers for RA patients' behavior toward their medication. Decisions not to take DMARDs were driven by the perception that RA was a nonserious, short-term condition, having insufficient information regarding the disease and the importance of regular DMARD therapy, and lack of discussion around disease parameters (eg, blood-based inflammatory marker results and disease activity scores). The high-adhering patients based their decision to take DMARDs on the perception that RA was a serious and long-term disease and felt that they had received sufficient information regarding the disease and disease activity parameters.

In line with other studies, the findings in our study showed the extent to which patient beliefs influenced treatment adherence - this has been widely reported in other chronic conditions, including asthma, cardiac disease, renal failure, cancer, and human immunodeficiency virus. ${ }^{14,15,26}$ The NCF and SRM work suggests that a patient's adherence decisions are a result of the balance between their perceived need for the medication (necessity) and their concerns regarding its use. We were able to explore how patients with RA balanced these to make decisions about DMARDs. Patients in our study who adhered well recognized the necessity of DMARD therapy, but some low-adhering South Asian patients clearly had doubts about their personal need for treatment. Studies on DMARD adherence, as well as studies on other conditions have also shown similar importance of medication beliefs. ${ }^{27-29}$ In our study, nonadherence in RA, therefore, was affected by individuals' views about perceived illness.

Due to symptoms being episodic, the low-adhering patients tended to view their RA as being short term and nonserious. This was particularly evident in the South Asian patients. Similarly, a study conducted in India also found that South Asian patients did not regard RA as a serious condition and had negative beliefs about RA treatments. ${ }^{30}$ Similar concepts have been reported among South Asian patients with other chronic conditions. ${ }^{31-34}$ In our study, South Asian patients with RA suggested that awareness of RA within their community and of the medication used for its management had an influence on their adherence to DMARDs. Lack of knowledge of RA and delays in initiating treatment have been reported in our previous work. ${ }^{35,36}$ Furthermore, a study conducted in India ${ }^{30}$ reported that South Asian patients with RA were more familiar with traditional remedies than Western medicines, and were found to be using these remedies alongside Western medicines. Similarly, our study has also found that some patients viewed traditional remedies favorably and felt that these could help to improve their RA symptoms.

Our study has identified potentially modifiable patient beliefs. Clinicians need to address patients' perceptions of medication need and concerns early in the treatment course. The key messages from our data were that the need for 
treatment and decisions to continue taking DMARDs were reinforced by receiving adequate information and exposure to visual representations of the disease process, particularly images of their own joints obtained by ultrasound or plain radiography. Evidence regarding improving patients' experiences or understanding of the disease by ultrasound scanning is not well documented in RA. ${ }^{37}$ To date, one pilot study of 18 patients with RA has suggested that using ultrasound to illustrate the processes operating in their joints can reduce patients' concerns about treatment. ${ }^{37}$ Furthermore, the study showed that using ultrasound scans as an educational tool helped to increase beliefs about the necessity of treatment. However, with its small sample size and short follow-up, the study was not designed to measure the effect of this intervention on medication adherence levels. Larger studies are required to investigate the role of ultrasound scanning in improving medication adherence in RA. The findings of our study suggested that apart from visualization of joints, the ability to view graphical plots of clinical parameters such as the disease activity score and levels of inflammatory markers may be helpful in improving patients' understanding of the need for long-term therapy.

The utility of pictorial/visualization tools to engage patients in understanding their disease and its treatment and in reducing anxiety has been reported in other conditions. For example, in relation to ovarian cancer, it has been shown that the use of ultrasound scanning of the ovaries reduced patients' anxiety and improved their confidence and well-being. ${ }^{38}$ Furthermore, the use of a moving threedimensional heart in the context of explanation of future risks of heart disease had more impact on patients than did text-based information alone. ${ }^{39,40}$ The authors noted that the three-dimensional images of the heart increase patients' common sense understanding of heart disease risk, causal beliefs, and control beliefs. Moreover, patients made more behavioral lifestyle changes, such as eating a healthy diet and taking up physical exercise, in response to such images. ${ }^{17}$ In a separate study ${ }^{40}$ that demonstrated change in behavior, the authors reported that an intervention, whose development was informed by components of the CSM, enhanced patients' understanding of heart disease risk and had a motivational effect on patient engagement. Building similar interventions in rheumatology might benefit patients from ethnic backgrounds such as those of South Asian origin who may need to be educated more about the differences between "curing" and "treating" RA with medicines and may also be of particular value in improving overall clinical outcomes in all patients.
This study had a number of limitations. Firstly, patients' views about other medicines that they were taking were not explored. Data regarding patients' views about medications such as antihypertensives might have provided insights regarding their relative views about different classes of drugs used to treat different conditions. The data from this study suggested that patients with RA expressed different views about conventional and biological DMARDs based on their experiences of the relative efficacy of these classes of medications. These views might have an effect on adherence; however, this aspect was not fully explored. The views of patients who refused to take part could have been different to those presented in this study. Despite these limitations, the strength of this study was that it captured views about medication adherence from adherent and nonadherent patients.

\section{Conclusion}

This study provides a deeper understanding of adherence to DMARDs in patients with RA. Improving adherence is likely to be facilitated by incorporating visual representations of the disease process, open discussion of disease parameters, and better explanations of the consequences of poorly controlled RA into the consultation. A good relationship with health professionals may also promote patients' adherence to DMARDs.

\section{Acknowledgments}

We would like to thank Amandeep Johal for reading and verifying the non-English transcripts. This study was funded by the National Institute for Health Research grant number (RGKP15291).

\section{Author contributions}

All authors contributed toward data analysis, drafting and revising the paper and agree to be accountable for all aspects of the work.

\section{Disclosure}

The authors report no conflicts of interest in this work.

\section{References}

1. Emery P. Evidence supporting the benefit of early intervention in rheumatoid arthritis. J Rheumatol Suppl. 2002;29(2):3-8.

2. National Audit Office Report. Services for people with rheumatoid arthritis. Available from: http://www.nao.org.uk/publications/0809/ rheumatoid_arthritis.aspx. Accessed April 7, 2015.

3. Kitas GD, Gabriel SE. Cardiovascular disease in rheumatoid arthritis: state of the art and future perspectives. Ann Rheum Dis. 2011;70(1):8-14.

4. Young A, Dixey J, Kulinskaya E, et al. Which patients stop working because of rheumatoid arthritis? Results of five years' follow up in 732 patients from the Early RA Study (ERAS). Ann Rheum Dis. 2002;61(4):335-340. 
5. Symmons DP, Prior P, Scott DL, Brown R, Hawkins CF. Factors influencing mortality in rheumatoid arthritis. J Chronic Dis. 1986;39(2):137-145.

6. Pasma A, Van't Spijker A, Hazes JM, Busschbach J, Luime J. Factors associated with adherence to pharmaceutical treatment for rheumatoid arthritis patients: a systematic review. Semin Arthritis Rheum. 2013; 43(1): $18-28$.

7. World Health Organization. Adherence to Long-Term TherapiesEvidence for Action. Geneva: World Health Organization; 2003.

8. Osterberg L, Blaschke T. Adherence to medication. $N$ Engl J Med. 2005;353(4):487-497.

9. Martin LR, Williams SL, Haskard KB. The challenges of patient adherence. Ther Clin Risk Manag. 2005;1(3):189-199.

10. van den Bemt BJ, Zwikker HE, van den Ende CH. Medication adherence in patients with rheumatoid arthritis: a critical appraisal of the existing literature. Expert Rev Clin Immunol. 2012;8(4):337-351.

11. Neame R, Hammond A. Beliefs about medications: a questionnaire survey of people with rheumatoid arthritis. Rheumatology (Oxford). 2005;44(6):762-767.

12. Goodacre LJ, Goodacre JA. Factors influencing the beliefs of patients with rheumatoid arthritis regarding disease-modifying medication. Rheumatology (Oxford). 2004;43(5):583-586.

13. Horne R. Patients' beliefs about treatment: the hidden determinant of treatment outcome? J Psychosom Res. 1999;47(6):491-495.

14. Horne R, Weinman J. Self regulation and self management in asthma: exploring the role of illness perceptions and treatment beliefs in explaining non-adherence to preventor medication. Psychol Health. 2002; 17:17-32.

15. Horne R, Weinman J. Patients' beliefs about prescribed medicines and their role in adherence to treatment in chronic physical illness. J Psychosom Res. 1999;47(6):555-567.

16. Clifford S, Barber N, Horne R. Understanding different beliefs held by adherers, unintentional nonadherers, and intentional nonadherers: application of the Necessity-Concerns Framework. J Psychosom Res. 2008;64(1):41-46.

17. Leventhal H, Nerenz DR, Steele DJ. Illness representations and coping with health threats. In: Taylor SE, Singer JE, editors. Handbook of Psychology and Health. Hillsdale, NJ: Erlbaum; 1984;45(4):219-252.

18. Kumar K, Gordon C, Barry R, Shaw K, Horne R, Raza K. 'It's like taking poison to kill poison but I have to get better': a qualitative study of beliefs about medicines in rheumatoid arthritis and systemic lupus erythematosus patients of South Asian origin. Lupus. 2011;20(8):837-844

19. Kumar K, Gordon C, Toescu V, et al. Beliefs about medicines in patients with RA and SLE: a comparison between patients of South Asian and White British origin. Rheumatology. 2008;47(5):690-697.

20. Cooper V, Gellaitry G, Hankins M, Fisher M, Horne R. The influence of symptom experiences and attributions on adherence to highly active anti-retroviral therapy (HAART): a six-month prospective, follow-up study. AIDS Care. 2009;21(4):520-528.

21. Kumar K, Raza K, Nightingale P, et al. A mixed methods protocol to investigate medication adherence in patients with rheumatoid arthritis of White British and South Asian origin. BMJ Open. 2013;3:1-6.

22. Arnett FC, Edworthy SM, Bloch DA, et al. The American Rheumatism Association 1987 revised criteria for the classification of rheumatoid arthritis. Arthritis Rheum. 1988;31(3):315-324.

23. Greenhalgh T, Taylor R. Papers that go beyond numbers (qualitative research). BMJ. 1997;315(7110):740-743.
24. Dixon-Woods M, Agarwal S, Jones D, Young B, Sutton A. Synthesising qualitative and quantitative evidence: a review of possible methods. J Health Serv Res Policy. 2005;10(1):45-53.

25. Hewitt-Taylor J. Use of constant comparative analysis in qualitative research. Nurs Stand. 2001;15(42):39-42.

26. Horne R, Cooper V, Gellaitry G, Date HL, Fisher M. Patients' perceptions of highly active antiretroviral therapy in relation to treatment uptake and adherence: the utility of the necessity-concerns framework. J Acquir Immune Defic Syndr. 2007;45(3):334-341.

27. de Thurah A, Norgaard M, Harder I, Stengaard-Pedersen K. Compliance with methotrexate treatment in patients with rheumatoid arthritis: influence of patients' beliefs about the medicine. A prospective cohort study. Rheumatol Int. 2010;30(11):1441-1448.

28. Treharne GJ, Lyons AC, Kitas GD. Medication adherence in rheumatoid arthritis: effects of psychosocial factors. Psychol Health Med. 2004;9(2):337-349.

29. Horne R, Chapman SC, Parham R, Freemantle N, Forbes A, Cooper V. Understanding patients' adherence-related beliefs about medicines prescribed for long-term conditions: a meta-analytic review of the necessity-concerns framework. PLoS One. 2013;8(12):e80633.

30. Chitnis N, Metha PN, Samant R, Bhojani KS, Joshi VR. Lag time between disease onset and first visit to a tertiary rheumatology centre by patients with rheumatoid arthritis. INJR. 2013;8:161-164.

31. Lawton J, Ahmad N, Peel E, Hallowell N. Contextualising accounts of illness: notions of responsibility and blame in white and South Asian respondents' accounts of diabetes causation. Sociol Health Illn. 2007;29(6):891-906

32. Lawton J, Parry O, Peel E, Douglas M. Diabetes service provision: a qualitative study of newly diagnosed type 2 diabetes patients' experiences and views. Diabet Med. 2005;22(9):1246-1251.

33. Lawton J, Ahmad N, Hanna L, Douglas M, Hallowell N. 'I can't do any serious exercise': barriers to physical activity amongst people of Pakistani and Indian origin with type 2 diabetes. Health Educ Res. 2006;21(1):43-54.

34. Kishore J, Ahmad I, Kaur R, P K M. Beliefs and perceptions about cancers among patients attending radiotherapy OPD in Delhi, India. Asian Pac J Cancer Prev. 2008;9(1):155-158.

35. Kumar K, Daley E, Carruthers DM, et al. Delay in presentation to primary care physicians is the main reason why patients with rheumatoid arthritis are seen late by rheumatologists. Rheumatology (Oxford). 2007; 46(9): 1438-1440.

36. Kumar K, Daley E, Khattak F, Buckley CD, Raza K. The influence of ethnicity on the extent of, and reasons underlying, delay in general practitioner consultation in patients with RA. Rheumatology (Oxford). 2010; 49(5):1005-1012.

37. El Miedany, El Gaafary M, Palmer D. Assessment of the utility of visual feedback in the treatment of early rheumatoid arthritis patients: a pilot study. Rheumatol Int. 2012;32(10):3061-3068.

38. Tadmor OP, Zlotogorski Z, Galron-Duniec M, et al. The effect of feedback on anxiety levels during ultrasound scanning for ovarian cancer. Ultrasound Obstet Gynecol. 1995;6(2):135-139.

39. Shahab L, Hall S, Marteau T. Showing smokers with vascular disease images of their arteries to motivate cessation: a pilot study. Br J Health Psychol. 2007;12(2):275-283.

40. Bovet P, Perret F, Cornuz J, Quilindo J, Paccaud F. Improved smoking cessation in smokers given ultrasound photographs of their own atherosclerotic plaques. Prev Med. 2002;34(2):215-220.
Patient Preference and Adherence

\section{Publish your work in this journal}

Patient Preference and Adherence is an international, peer-reviewed, open access journal that focuses on the growing importance of patient preference and adherence throughout the therapeutic continuum. Patient satisfaction, acceptability, quality of life, compliance, persistence and their role in developing new therapeutic modalities and compounds to optimize

\section{Dovepress}

clinical outcomes for existing disease states are major areas of interest for the journal. This journal has been accepted for indexing on PubMed Central The manuscript management system is completely online and includes a very quick and fair peer-review system, which is all easy to use. Visit http://www. dovepress.com/testimonials.php to read real quotes from published authors. 